\title{
IATROGENIC ABERRATIONS OF SERUM ENZYME ACTIVITY*
}

\author{
J. G. BATSAKIS, J. A. PRESTON, R. O. BRIERE, and P. C. GIESEN \\ Department of Pathology, University of Michigan Medical Center, Ann Arbor, Michigan \\ (Drs. Batsakis and Giesen), The Colorado Pathologists Reference Laboratory, \\ Denver, Colorado (Dr. Preston) and the Johnston-Willis Hospital, \\ Richmond, Virginia (Dr. Briere)
}

(Received A ugust 26, 1968)

\section{SUMmary}

1. Three forms of iatrogenic serum enzyme alteration are presented, each entailing a different mechanism of action.

2. Recent modifications in creatinine phosphokinase (CPK) assay systems have greatly increased the sensitivity of this measurement. This increase in sensitivity has been accompanied by a relative decrease in clinical specificity. In patients receiving intramuscular injections of drugs, resort may have to be made to the SGOT, LDH or isoenzymes of $\mathrm{LDH}$. The latter also appear to be the only common measurements not liable to modification by narcotics injected intra-muscularly in patients with biliary tract disease.

3. Post-valvulotomy patients with anginal chest pain cannot be satisfactorily assessed by LDH or LDH isoenzyme assays. CPK and SGOT estimations are the procedures of choice because of their relative insusceptibility to activity changes secondary to hemolysis.

ENZYME ACTIVITY ESTIMATED IN THE SERA of patients is a quantification affected by a variety of in vivo and in vitro phenomena.

Figure 1 depicts some of the variables capable of modifying the quantification of serum enzyme activity. These variables combined with the absence of an organ-specific enzyme underline the relative empiricism of clinical enzymology.

Conceding the responsibility of the clinical laboratory to correctly perform, control and report the enzyme estimation, there exist a number of iatrogenic alterations which have a direct influence on the clinical interpretations of the reported enzyme activity.

Modifications of serum enzyme activity by exercise, surgical procedures, co-existent disease, or by a host of in-laboratory variables have been repeatedly

Correspondence to Dr. Batsakis, 1335 E. Catherine Street, Ann Arbor, Michigan 48104.

*This paper was supported by a grant from the Michigan Heart Association. 


\section{FACTORS INFLUENCING MEASURED SERUM ENZYME ACTIVITY}

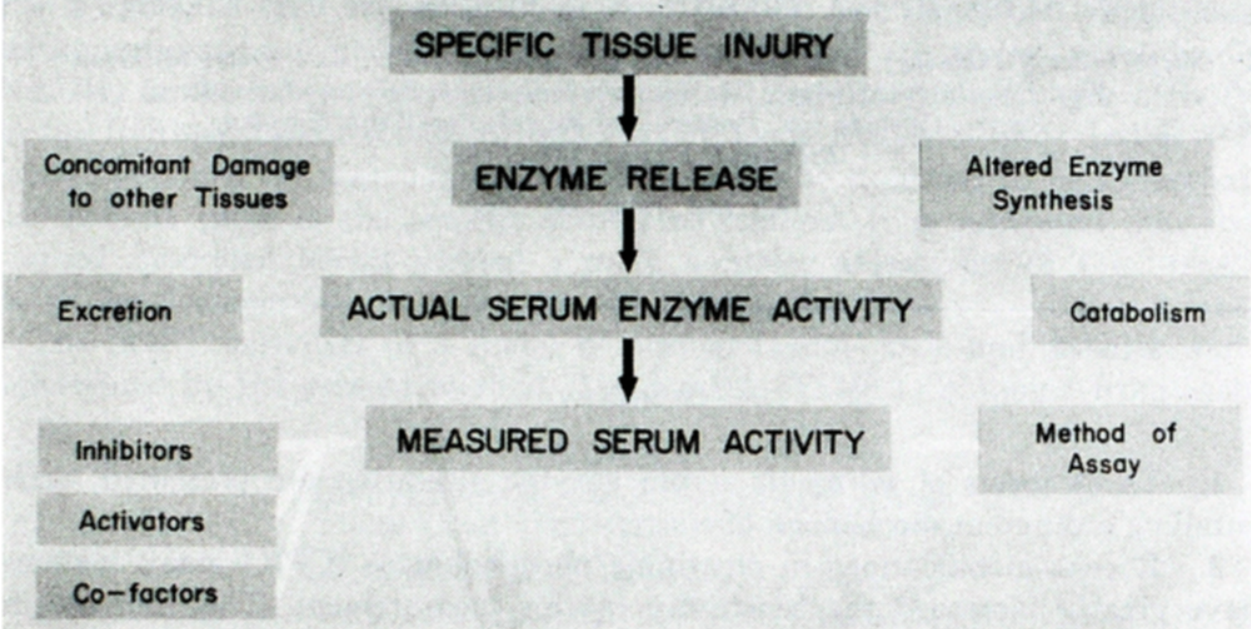

FIG. 1

documented in the literature. Relatively little information concerning iatrogenic aberrations has been reported. In the instance of myocardial infarct, the differential diagnosis may be so beclouded by spurious rises in serum enzyme activity that (1) surgical procedures are postponed or (2) inappropriate medical therapy is instituted.

This report presents three forms of iatrogenic alterations in serum enzyme activity as they relate to the diagnosis of acute myocardial infarct in the pre- or postoperative patient. The spurious enzyme activity in each is initiated by a different mechanism and is unrelated to surgical manipulation of tissues.

\section{Opiate-induced hypertransaminasemia}

The administration of opiates (morphine, codeine, mepheridine) in normal subjects is followed by a rise in the serum activities of both amylase and lipase (1). Induced constriction of the sphincter of Oddi and a resultant increase in intraductal pressure within the pancreatic ducts is responsible for the rise in activity. The opiate-induced rise in serum activity is not striking and should not give rise to serious confusion in the differential diagnosis of pancreatitis. On the other hand, considerable confusion may be engendered by the use of opiates in select patients suspect for myocardial infarct.

Opiates, through the aforementioned action, also produce a rise in intrabiliary tract pressure and this may elicit not only pain in the chest or abdomen, but also electrocardiographic alterations (2). Some subjects also manifest a rise in their 
transaminase (SGOT) and lactic dehydrogenase ( $\mathrm{LDH}$ ) activities as a consequence of this increased pressure. The magnitude of the rise, particularly with the SGOT, may be equal to or greater than that expected after an acute myocardial infarct $(2,3)$.

The iatrogenic changes of serum enzyme activity appear to be limited to patients with a diseased biliary tract or to those who have undergone an earlier cholecystectomy (3). It is this category of patients who lack a "reservoir of functional safety valve," i.e., the gallbladder, to absorb the added intraductal pressure. No significant increase in either the transaminase or LDH occurs in healthy normals with an intact biliary tract system after the administration of opiates (4).

The combination of alterations in serum enzyme activity, chest pain and potential spurious electrocardiographic changes may lead to an erroneous diagnosis of myocardial infarct. Figure 2 is representative of the SGOT alterations capable of being induced in this group of patients. In six patients studied, the SGOT generally began to be significantly elevated at two to four hours after the

\section{PSEUDO-MYOCARDIAL INFARCT SYNDROME (PATIENTS WITH BILIARY TRACT DISEASE)}

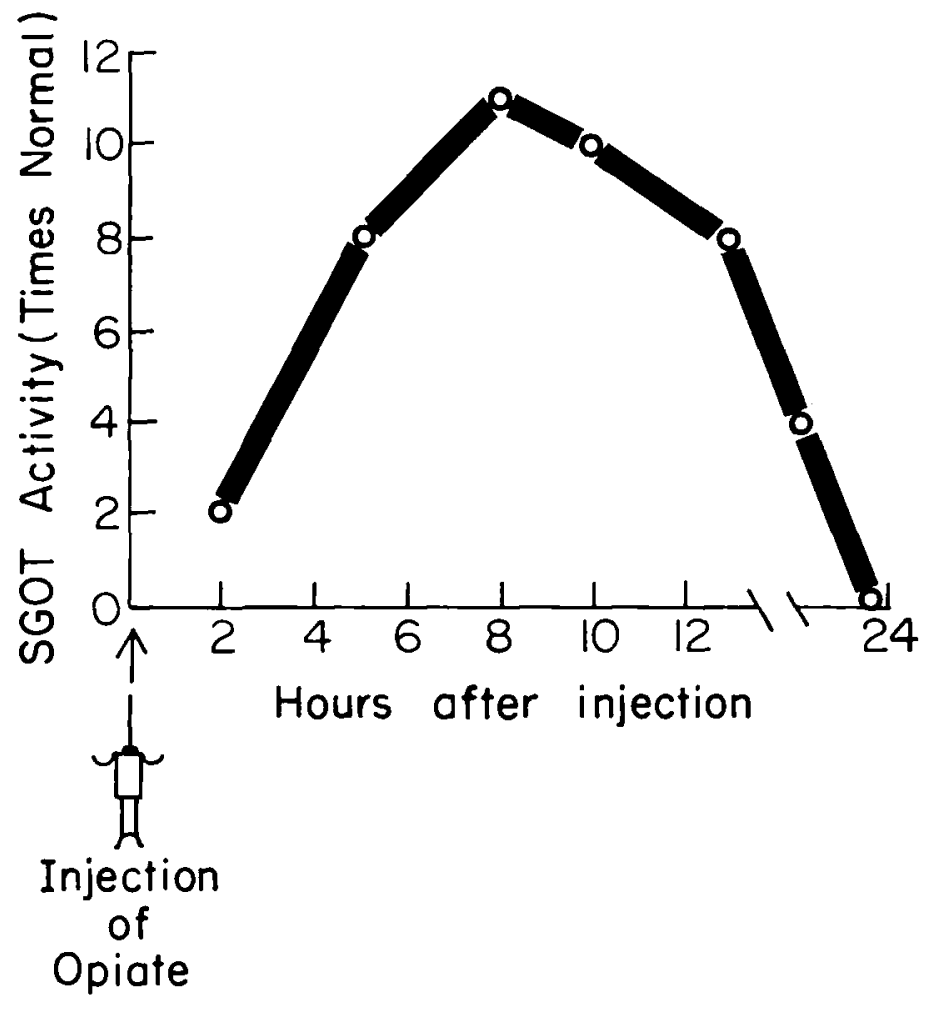

FIG. 2 
use of opiates. Peak activities were observed at five to eight hours and abnormal activity in the sera persisted for as long as 24 hours after the use of the drug. Transaminase increments ranged from two and one-half to 65 times control levels.

Because of the potential inability of the serum SGOT and LDH to assist in differential diagnosis, concomitant estimation of creatine phosphokinase (CPK) activity is advised. A high degree of clinical judgement is nevertheless required, particularly so since the latter measurement is also subject to iatrogenic alterations.

\section{Sensitized CPK assay systems}

The second form of iatrogenic elevation of serum enzyme activity does not require the absence of a "safety valve" for its cause, but is directly related to recent modifications in the sensitivity of assay systems for the estimation of creatine phosphokinase (CPK) activity.

In 1965, the present authors (5) and others reported on the clinical application of CPK estimations for assistance in the differential diagnosis of acute myocardial infarcts and myopathic disorders. Certain "disadvantages" however, were apparent with the then-available assay systems. Normal sera had low levels of enzyme activity and variable rates of "inactivation" of the enzyme were reported. Special precautions also had to be taken for the detection of the female heterozygote of muscular dystrophy.

Two recent developments have largely corrected these "disadvantages" and have thereby increased the sensitivity of assay systems.

CPK catalyzes the reversible formation of adenosine triphosphate (ATP) and creatinine from adenosine diphosphate (ADP) and creatine phosphate according to the equation:

$$
\text { ADP }+ \text { creatine phosphate } \rightleftharpoons \text { ATP + creatine. }
$$

The activity of the enzyme can be estimated by utilizing methods involving both directions of the equation.

Increased sensitivity of CPK estimations is acknowledged to accompany utilization of the forward reaction of the equation and this is widely used by clinical laboratories $(6,7)$.

The second step towards increasing the sensitivity appears to have been an outgrowth of biochemical attempts to combat the loss of enzyme activity upon storage of serum samples.

Inactivation of enzymes containing thiol groups in their active centers may be prevented by addition of cysteine or glutathione with the former more widely used $(7,8)$. "Activation" appears to be due to a form of de-blocking of thiol groups of the enzyme molecules by added thiol groups.

Regardless of the direction of the reaction used to estimate CPK activity, the observed enhancement of activity after the addition of cysteine appears to be a reactivation of the enzyme and not an activating effect on a still fully active enzyme. The fact that Weismann et al. (8) did not observe a change in the Michae- 
lis constant for creatine and ATP by the addition of cysteine supports this interpretation.

Whatever the mechanism, added thiols produce a three- to tenfold increase in CPK activity of normal sera and this combined with the more sensitive forward reaction further increases sensitivity $(7,8)$. To our knowledge all commercially available reagent systems for the estimation of CPK activity include some form of thiol enhancement.

The reagent system* used in the present study has been recommended as the most sensitive, most convenient and least time-consuming of any other currently available procedure for the measurement of CPK activity ( $(7)$.

Nothing evolved from this study contradicts this statement, but the use of enhanced or stimulated assay systems does pose an additional element of clinical judgement for the interpretation of elevated CPK activity in body fluids. This relates almost exclusively to the heightened sensitivity of the reaction and to the relative specificity of $\mathrm{CPK}$, i.e., skeletal and cardia muscle and central nervous system tissue (5).

In patients sustaining acute myocardial infarcts, documented by electrocardiographic and/or other serum enzyme elevations, an increase in serum CPK activity is demonstrable as early as three to six hours after the clinical onset of chest pain. The time-reaction curve for our group of patients with infarcts is illustrated in Figure 3. The early rise of $\mathrm{CPK}$, of ten preceding changes in other enzyme activities, has also been reported for CPK assay systems not using the stimulated reaction and does not represent a change (j).

Persistence of elevated serum activity for as long as eight days after uncomplicated infarct, however, does not constitute a significant difference from experience using the older non-stimulated reactions (5). With the latter normal activity was achieved by the end of the third day after infarct.

We also hold the sensitized CPK reaction systems responsible for the finding of elevated activity in patients without infarct but with cardiac arrhythmias; an observation not noted in earlier reports before the introduction of cysteine enhancement. Twelve consecutive patients, in our series, manifesting ventricular rates of $160 / 240$ minute, demonstrated a rise in their serum CPK activity of between 66-98 IU. Twenty-four hours after restoration to a normal rate of rhythm, the serum CPK was also normal.

While the foregoing represent direct outgrowths of thiol stimulation on the enzymatic reaction used to estimate CPK activity, they rarely serve as a source of confusion to the clinician. The effect of intramuscular injections, however, on $\mathrm{CPK}$ activity measured by the enhanced reaction assays, is sufficiently constant that it is now common practice in our respective laboratories to investigate whether or not intramuscular injections have been administered before clinical significance is attached to the quantification of enzyme activity.

Hess and MacDonald (9) evaluating a non-cysteine stimulated reaction for measurement of $\mathrm{CPK}$, reported muscle trauma, particularly extensive surgery or

${ }^{*}$ Reagents available in kit form (CPK Capsule), Calbiochem Inc., Los Angeles, California. 


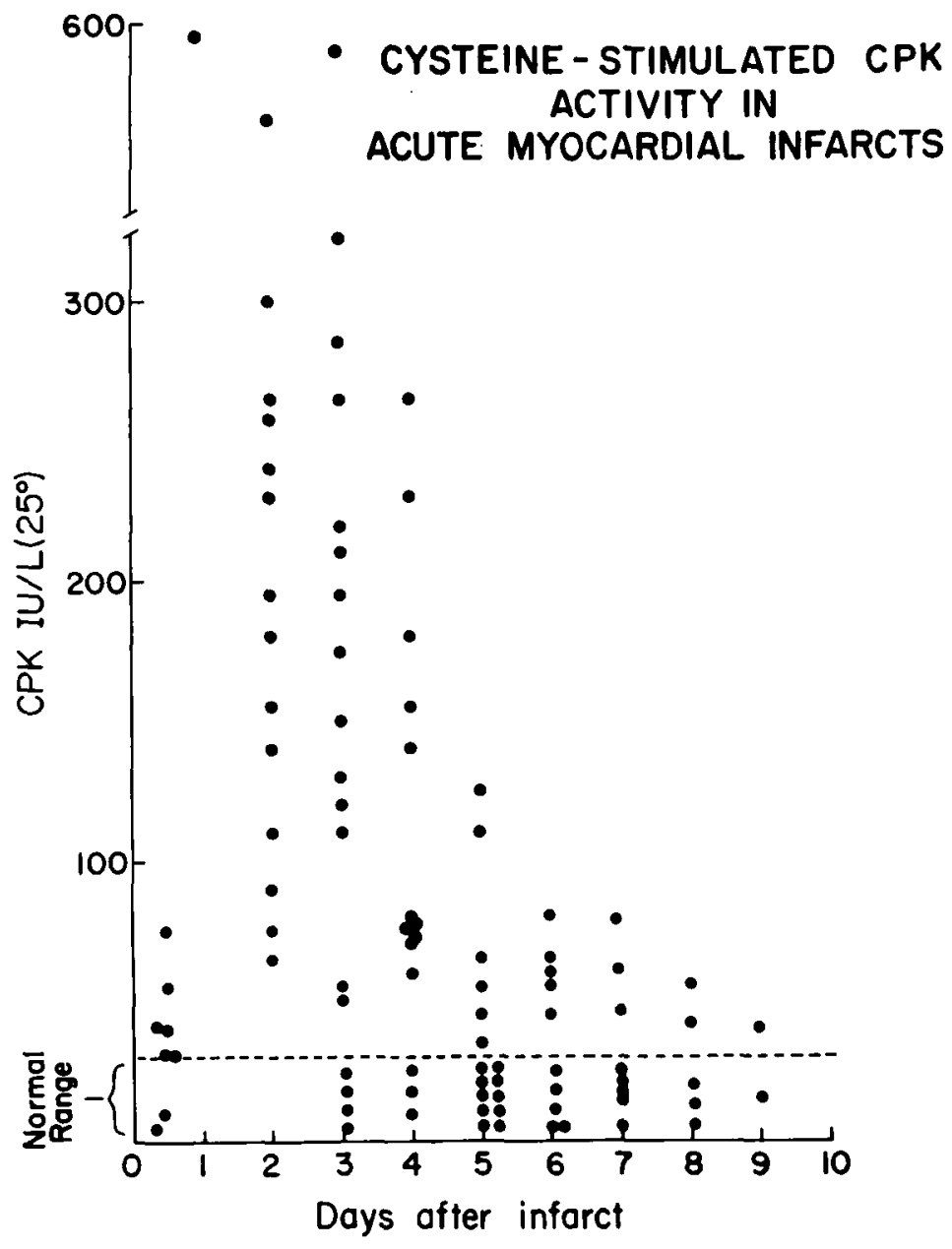

FIG. 3

crushing injuries, produced a rise in serum CPK activity. Preston et al. (j) found a similar sequence and related the height of activity to the extent of muscle injury. Hess and MacDonald (9) further indicated that frequent intramuscular injections of irritating drugs such as aqueous penicillin were also capable of producing a rise in serum CPK activity up to four or five times normal. They claimed narcotics, barbiturates and diuretics, administered by the same route, had no detectable influence.

Table 1 indicates the serum CPK activity observed following the intramuscular injection of drugs in our series of patients studied with the aid of a thiol-stimulated CPK reaction. Note the increased activity is not limited to either frequent or to "irritating" drugs. The highest activity was observed in a subject receiving multiple injections of diuretics, antibiotics and analgesics, indicating the effects may be additive. Three additional patients are depicted in Figure 4. The patients 
TABLE 1

Serum CPK Activity in Response to Intramuscular Injections

\begin{tabular}{lcc}
\hline \hline \multicolumn{1}{c}{ Drug Frequency } & No. of Patients & CPK Activity (I.U.) \\
\hline Penicillin, every 4 hrs. & 6 & $60-200$ \\
Morphine, every 4-6 hrs. & 4 & $65-98$ \\
Morphine + diuretic, every & 4 & $70-130$ \\
6-12 hrs. & 2 & $67-98$ \\
Multiple analgesics & 2 & $160-240$ \\
Narcotics, diuretics & & \\
penicillin, every 6-8 hrs. & &
\end{tabular}

\section{EFFECT OF INTRA-MUSCULAR INJECTIONS ON SERUM CPK ACTIVITY}

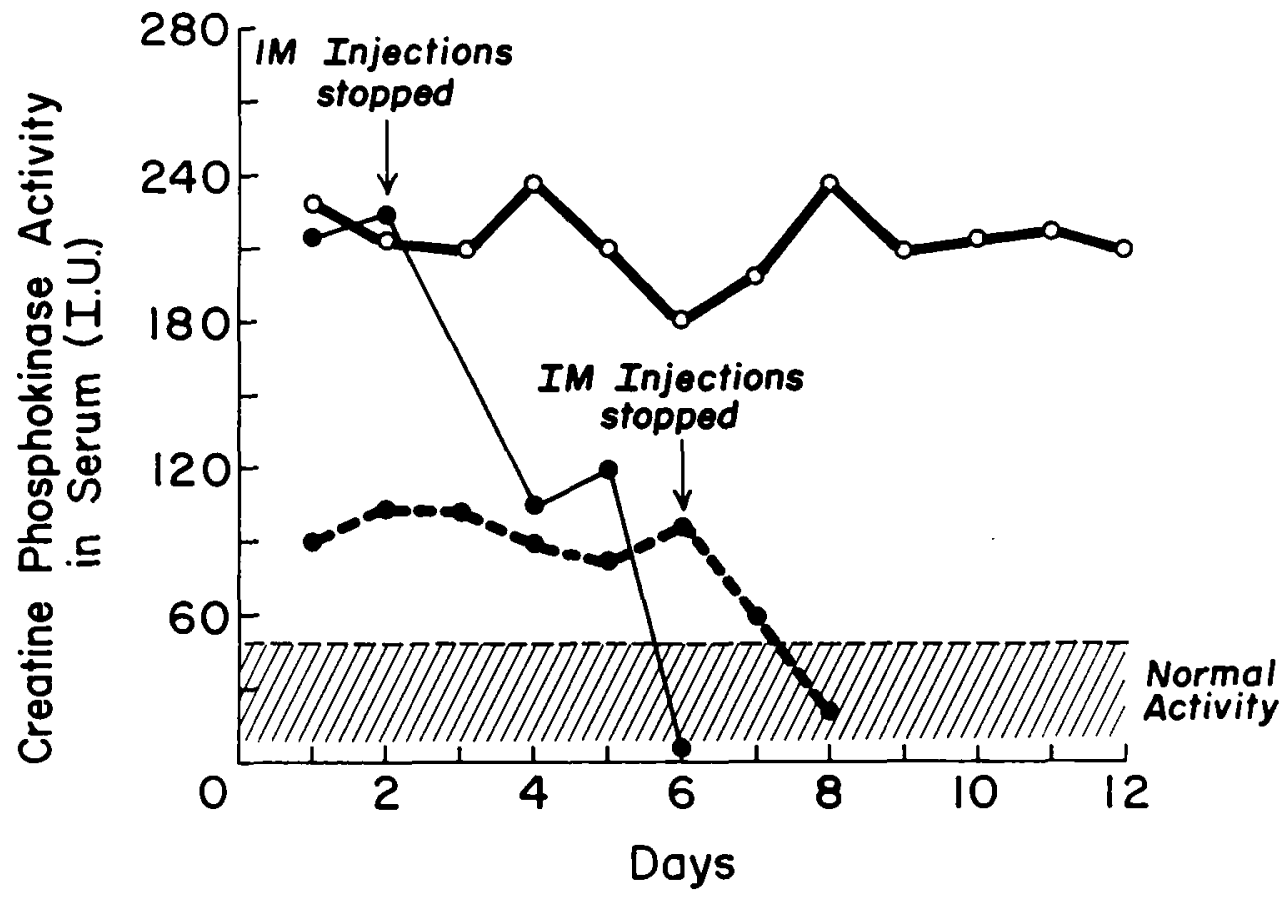

FIG. 4

were followed with serial CPK estimations for an extended time. The relative constancy of the activity, combined with the absence of a concomitant rise in the SGOT and LDH over such a period of time serve as indicators that intramuscular injections are responsible for the rise in CPK activity. The return to normal levels of activity in patients receiving spaced injections of no more than two drugs is usually achieved within 48 hours after injections have stopped. 


\section{Post-cardiac surgery aberrations}

Hemolysis associated with intracardiac prosthetic material has been reported for the past decade. In the immediate postoperative period, the mechanics of cardiopulmonary bypass procedure are held responsible for the observed lack of haptoglobin and methemalbumin and an elevated LDH activity in the patient's sera. The mechanical procedure, however, cannot be held responsible for the persistence of these biochemical abnormalities after many months following the surgical procedure.

A number of reports now indicate a chronic hemolytic process, of variable severity, is in fact a consistent finding in patients in whom a prosthetic cardiac valve has been placed $(10,11)$. Data from eleven patients with valve prostheses (Table 2) are in accordance with these reports. Jorgensen et al. (10) have recorded

TABLE 2

Valve Prostheses and LDH Activity in Serum

\begin{tabular}{|c|c|c|c|c|}
\hline \multirow[b]{2}{*}{ Site of Prosthetic Device } & \multirow[b]{2}{*}{ Postoperative Time I.apse } & \multicolumn{3}{|c|}{ LDH Activity* } \\
\hline & & Total & - & Isoenzymes \\
\hline Mitral & 2 mos. & 90 & & $\mathrm{OH}_{1} \& \mathrm{LDH}_{2}$ \\
\hline Mitral & 3 mos. & 92 & & , \\
\hline Mitral & 5 mos. & 120 & & $"$ \\
\hline Mitral & 10 mos. & 98 & & $"$ \\
\hline Mitral & 11 mos. & 87 & & ." \\
\hline Mitral & 20 mos. & 110 & & ", \\
\hline Mitral & 30 mos. & 95 & & "., \\
\hline Mitral & 36 mos. & 105 & & " \\
\hline Aortic & 6 mos. & 100 & & ", \\
\hline Aortic & 5 mos. & 100 & & ", \\
\hline Aortic & $3 \mathrm{mos}$ & 98 & & ". \\
\hline
\end{tabular}

*Upper limit of normal equals 77 I.U.

almost identical observations. These workers found: (1) a uniform elevation of total LDH activity and (2) a uniform increase of the heat-stable $\left(\mathrm{LDH}_{1}\right.$ and $\mathrm{LDH}_{2}$ ) isoenzymes in all patients bearing various prosthetic incardiac devices from one month to almost five years after surgery.

As indicated by Bell and his associates (11) it seems unlikely the hemolysis associated with the prosthetic device is solely attributable to the presence of the foreign material. Valve incompetence and a turbulent jet effect may appear to be the primary factors in the production of hemolysis. An additional nine patients studied by the present authors support this premise. Five of the nine patients with severe aortic valve disease manifested an elevated total LDH activity and predominance of $\mathrm{LDH}_{1}$ and $\mathrm{LDH}_{2}$ by electrophoresis before attempted surgical correction of the valvular disorder. Similar findings have been reported by others $(10,11)$. Data thus far accumulated suggest that all patients with prosthetic valves and a significant number of patients with uncorrected severe hemodynamic abnormalities have a chronic, usually compensated, intravascular hemolysis related to damaged erythrocytes.

The importance of these findings relate to the obviation of the total LDH activity and isoenzymes of LDH for the confirmation of myocardial infarct for 
the life of these patients. The liberation of erythrocytic $\mathrm{LDH}$ with its predominantly fast isoenzymes $\left(\mathrm{LDH}_{1}\right.$ and $\left.\mathrm{LDH}_{2}\right)$ so alters the serum configuration of the enzyme and its isoenzymes that other enzymatic indices must be used for evaluating chest pain in patients with cardiac valvular prostheses or with congenital or acquired valvular disease.

\section{REFERENCES}

1. Gross, J. B., Comfort, M. W., Mathieson, O. R. \& Power, M. H. Elevated values of serum amylase and lipase following administration of opiates. Proc. Staff Meet. Mayo Clinic 28, 81-87 (1951).

2. Mossberg, S. M., Bloom, A., Berkowitz, J. \& Ross, G. Serum enzyme activities following morphine: a study of transaminase and alkaline phosphatase levels in normal persons and those with gall-bladder disease. Arch. Intern. Med. 109, 429-437 (1962).

3. Batsakis, J. G. \& Briere, R. O. Interpretive Enzymology, Springfield, Illinois, C. C. Thomas, 1967.

4. Shuster, F., Napier, E. A., Jr. \& Henley, K. S. Serum transaminase activity following mepherdine, morphine and codeine in normals. Amer. J. Med. Sci. 246, 714-716 (1963).

5. Preston, J. A., Batsakis, J. G., Briere, R. O. \& Taylor, R. V. Serum creatine phosphokinase-a clinical and laboratory evaluation. Amer. J. Clin. Path. 44, 71-76 (1965).

6. Nielsen, L. \& Ludvigsen, B. Improved method for determination of creatine kinase. J. Lab. Clin. Med. 62, 159-168 (1963).

$\tau$. Rosalki, S. B. An improved procedure for serum creatine phosphokinase determination. J. Lab. Clin. Med. 69, 696-705, (1967).

8. Wiesmann, U., Colombo, J. P., Adam, A. \& Richterich, R. Determination of cysteine activated creatine kinase in serum. Enzymol. Biol. Clin. 7, 266-284 (1966).

9. Hess, J. W. \& MacDonald, R. P. Serum creatine phosphokinase activity: a new diagnostic aid in myocardial and skeletal muscle disease. J. Mich. Med. Soc. 62, 1095-1099 (1963).

10. Jorgensen, C. R., Zimmerman, T. S. \& Wang, Y. Serum lactate dehydrogenase elevation in ambulatory cardiac patients: Evidence for chronic hemolysis. Circulation 35, 79-89 (1967).

11. Bell, R. E., Petuoglu, S. \& Fraser, R.S. Chronic hemolysis occurring in patients following cardiac surgery. Brit. Heart J. 29, 327-332 (1967). 